\title{
Association between Suicidal Ideation and Causes of Primary Care Visits: A Cross-Sectional Study to Identify Patients with Suspected Suicidal Ideation
}

\author{
Gyeongsil Lee ${ }^{1}$ and Jung-Ha Kim ${ }^{2}$ \\ ${ }^{1}$ Department of Family Medicine, Health Promotion Center, Seoul National University Hospital, Seoul, Republic of Korea \\ ${ }^{2}$ Department of Family Medicine, Chung-Ang University Hospital, Seoul, Republic of Korea
}

\begin{abstract}
Objective Suicide victims are likely to visit a primary care provider within 1 month of the suicide attempt. However, it is impossible for primary care providers to screen all patients for suicidal ideation or attempts. We, therefore, aimed to investigate potential differences in the chief diagnoses received by primary care patients with and without suicidal ideation, as this information may provide clues and predict patients at risk of suicide.

Methods This cross-sectional study included 1,211 Korean adults aged $\geq 20$ years who had participated in the Korea National Health and Nutrition Examination Survey during 2014 and provided information about their histories of suicidal ideation and visits to primary care providers. Multiple logistic regression analyses were used to examine whether suicidal ideation was associated with specific diseases after controlling for age, sex, household income, education, occupation, marital status, smoking, alcohol consumption, and physical activity.

Results Among primary care patients, the sample-weighted percentage of suicidal ideation was approximately $12 \%$. The diagnoses of neoplasm and mental disorder were associated with a higher likelihood of suicidal ideation \{odds ratio (OR) [95\% confidence interval (CI)] 8.32 (1.01-68.56) and 6.19 (1.72-22.33), respectively\}. Notably, diseases of the musculoskeletal system and connective tissue were also associated with suicidal ideation [OR (95\% CI): 1.93 (1.09-3.43)].

Conclusion The observed visit patterns and correlations of certain diagnoses with suicidal ideation suggest that primary care physicians should pay attention to patients with relevant disorders, consider screening suicidal ideation, and properly consult to a psychiatrist.
\end{abstract}

Psychiatry Investig 2020;17(7):667-673

Key Words Suicide, Mood disorder, Community psychiatry.

\section{INTRODUCTION}

Suicide is a critical global public health issue, ${ }^{1}$ particularly in the Republic of Korea where the prevalence of suicide is among the highest worldwide. According to a report from the Korea Suicide Prevention Center, the national prevalence of suicide increased steadily from 16.6 to 33.5 per 100,000 people during 2009-2010 and decreased slightly thereafter to 26.6 per 100,000 people in $2018 .{ }^{2}$ In contrast, the average prevalence of suicide among Organization for Economic Coopera-

\footnotetext{
Received: February 9, 2020 Revised: March 18, 2020

Accepted: April 7, 2020

$\triangle$ Correspondence: Jung-Ha Kim, MD, PhD

Department of Family Medicine, Chung-Ang University Medical Center, 102 Heukseok-ro, Dongjak-gu, Seoul 06973, Republic of Korea

Tel: +82-2-6299-1891, Fax: +82-2-6299-2064, E-mail: girlpower219@cau.ac.kr

(c) This is an Open Access article distributed under the terms of the Creative Commons Attribution Non-Commercial License (https://creativecommons.org/licenses/bync/4.0) which permits unrestricted non-commercial use, distribution, and reproduction in any medium, provided the original work is properly cited.
}

tion and Development (OECD) countries were 11.5 per 100,000 people in 2017. ${ }^{2}$ This discrepancy highlights the status of suicide as a public health crisis in Korea.

The high suicide mortality patterns have elicited a range of preventive strategies worldwide. Several previous reports have stressed comprehensive and integrated multidisciplinary approaches for the reduction of suicide and suicidal behaviour, ${ }^{3-6}$ among which gatekeeper training is regarded one of the most effective strategies. Gatekeepers are individuals who come into frequent contact with members of the community; this category includes primary, mental, and emergency health care providers; teachers and other school staff; community leaders and police officers; and other first responders. ${ }^{3}$ Some studies have reported that approximately $45 \%$ of suicide victims had contact with a primary care provider within 1 month of the suicide attempt, ${ }^{7}$ suggesting that primary care providers could play a prominent role in suicide prevention. ${ }^{8}$ However, it would be impossible for primary care physicians to screen 
all visiting patients for suicidal ideation or attempts, as the general population is not considered a high-risk group. The U.S. Preventive Services Task Force (USPSTF) also concluded that "the current evidence is insufficient to assess the balance of benefits and harms of screening for suicide risk in adolescents, adults, and older adults in primary care." In summary, the prevention of suicide would rely on the identification of patients who are likely to commit suicide.

The Korean National Health and Nutrition Examination Survey (KNHANES) includes data about suicidal ideation, primary care visit history, and information about diagnoses made during the most recent 2 -week period. These data could form the basis of an investigation of differences in the chief complaints and diagnoses of primary care patients with and without suicidal ideation. The results of such an investigation could subsequently facilitate the prediction of patients at risk of committing suicide. Therefore, this study aimed to determine the association between suicidal ideation and the primary care visit history, using data from the KNHANES in 2014.

\section{METHODS}

\section{Study participants}

This study was conducted according to the guidelines set forth in the Declaration of Helsinki. All procedures involving human subjects were approved by the Institutional Review Board of the Korea Centers for Disease Control and Prevention (2013-12EXP-03-5C). Written informed consent was obtained from all subjects.

This cross-sectional study used data from the KNHANES, a nationwide survey of non-institutionalized civilian residents of South Korea that has been conducted by the Korean Ministry of Health and Welfare since 1998. Briefly, this nationwide representative study uses a stratified, multistage probability sampling design to select household units. ${ }^{10}$ The KNHANES aims to evaluate the nutritional and health status of the general population and subsequently provide representative and reliable statistical public health data that can be used as a basis for health care policies.

This analysis was based on data obtained from 5,428 respondents to the 2014 survey who were aged $\geq 20$ years. From this initial population, we excluded the following individuals for the following reasons: no information about outpatient visits during the most recent 2 -week period ( $n=3,670)$; no visits to a primary care center $(\mathrm{n}=536)$; and missing data for the ninth question on the Patient Health Questionnaire (PHQ-9; $n=11$ ). This study included 1,211 Korean adults.

\section{Study variables}

The PHQ-9 is a reliable and valid screening tool used wide- ly to detect depressive disorders, including suicidal ideation, in the general population. ${ }^{11}$ The Korean version of the PHQ-9, which is also considered reliable and valid, was included in the KNHANES VI-2 survey. ${ }^{12}$ In this study, we defined a participant as having suicidal ideation if they provided a positive response (i.e., "yes") to the ninth item on the PHQ-9, which asked "Have you ever thought that you would be better off dead or of hurting yourself in the most recent 2 weeks?"

Diseases that served as the causes for visits to primary care providers were identified via diagnostic information and had been recorded by physicians according to the Tenth International Classification of Diseases (ICD-10). The following codes and classifications were applied: A00-B99, Certain infectious and parasitic diseases; C00-C97, Neoplasms; E00-E90, Endocrine, nutritional, and metabolic diseases; F00-F99, Mental and behavioral disorders; G00-G99, Diseases of the nervous system; H00-H59, Diseases of the eye and adnexa; H60H95, Diseases of the ear and mastoid process; 100-I99, Diseases of the circulatory system; J00-J99, Diseases of the respiratory system; K00-K93, Diseases of the digestive system; L00-L99, Diseases of the skin and subcutaneous tissue; M00-M99, Diseases of the musculoskeletal system and connective tissue; N00-N99, Diseases of the genitourinary system; O00-O99, Pregnancy, childbirth, and the puerperium; R00-R99, Symptoms, signs, and abnormal clinical and laboratory findings, not elsewhere classified; S00-T98, Injury, poisoning, and certain other consequences of external causes; and Z00-Z99, Factors influencing health status and contact with health services. We also identified self-reported underlying diseases, including hypertension, diabetes mellitus, dyslipidemia, stroke, cardiovascular disease (angina or myocardial infarction), depression, and cancer diagnosed by a medical doctor.

The potential confounders included age, sex, residence, household income, education, marital status, body mass index (BMI), smoking status, alcohol consumption, and physical activity. The residence was classified as urban or rural. Household income was classified into quartiles. Education status was classified into two categories: high school or lower and college or higher. Occupations were classified as follows: manager, professional, or office worker; service or sales; manual worker; and unemployed (e.g., housewife or student). The cohabitation status was classified as either living alone or living together. BMI was derived from the measured weight and height and reported in units of $\mathrm{kg} / \mathrm{m}^{2}$. Obesity was defined as a BMI of $\geq 25 \mathrm{~kg} / \mathrm{m}^{2}$ according to the current Asian obesity guidelines. ${ }^{13} \mathrm{~A}$ current smoker was defined as someone who had smoked $\geq 100$ cigarettes (5 packs) throughout his or her lifetime and continued to smoke at the time of the survey. A heavy drinker was defined as a person who consumed at least one bottle of soju (Korean distilled spirits) during a 1-week pe- 
riod. Regular physical activity was defined as participation in vigorous activity (e.g., running or mountaineering) for $\geq 75$ $\mathrm{min} /$ week or moderately intense activity (e.g., light swimming, badminton, or walking) for $\geq 150 \mathrm{~min} /$ week. ${ }^{14}$ Strength exercise was defined as the performance of exercise at least twice a week.

\section{Statistical analysis}

The survey procedures in SAS 9.2 (SAS Institute Inc., Cary, NC, USA) were applied to complex sampling designs. The characteristics of the study population are reported as means \pm standard errors (SEs) for continuous variables and as weighted percentages (\%) with SEs for categorical variables. We categorized participants into two groups according to suicidal ideation, using the ninth item of the PHQ-9. Differences in ICD10 codes among the two groups were analyzed using a weighted t-test and chi-square test. Multiple logistic regression analyses were used to examine the potential associations of suicidal ideation with specific diseases after controlling for age, household income, education, occupation, marital status, smoking, alcohol consumption, and physical activity. Statistical significance was set at a two-sided p-value $<0.05$.

\section{RESULT}

Table 1 presents the general characteristics of the study population, stratified by suicidal ideation. The sample-weighted frequency of suicidal ideation among primary care patients was $11.8 \%$. Patients who expressed suicidal ideation were more likely to be female, have low income and education levels, live alone, be unemployed, and be less likely to perform strength exercise. Table 2 presents information about disease diagnoses made by primary care providers during the 2 -week period before the survey according to ICD-10 codes and selfreported underlying diseases, according to the suicidal ideation status. Of the ICD-10 coded diseases, mental and behavioral disorders (F00-F99) and diseases of the musculoskeletal system and connective tissue (M00-M99) were significantly more frequent among individuals with suicidal ideation. Among self-reported underlying diseases, hypertension, stroke, and

Table 1. Participants' general characteristics $(\mathrm{N}=1,211)$

\begin{tabular}{|c|c|c|c|}
\hline & \multicolumn{2}{|c|}{ Suicidal ideation } & \multirow{2}{*}{ p-value } \\
\hline & No $(\mathrm{N}=1,057)$ & Yes $(\mathrm{N}=154)$ & \\
\hline$\%(\mathrm{SE})$ & $88.2(1.0)$ & $11.8(1.0)$ & \\
\hline Age (years) mean $\pm \mathrm{SE}$ & $51.1 \pm 0.7$ & $55.1 \pm 2.3$ & 0.08 \\
\hline Sex (men), \% (SE) & $42.4(1.6)$ & $24.1(4.6)$ & $<0.01$ \\
\hline Living (urban), \% (SE) & $82.9(2.8)$ & $77.0(4.5)$ & 0.11 \\
\hline Household income, \% (SE) & & & $<0.01$ \\
\hline Low & $17.0(1.6)$ & $46.5(5.2)$ & \\
\hline Lower-middle & $25.1(1.8)$ & $27.0(4.6)$ & \\
\hline Upper-middle & $30.7(2.0)$ & $9.4(2.7)$ & \\
\hline High & $27.1(2.3)$ & $17.1(4.5)$ & \\
\hline Education (college degree or higher), \% (SE) & $64.0(1.7)$ & $37.9(5.4)$ & $<0.01$ \\
\hline Occupation, \% (SE) & & & $<0.01$ \\
\hline Manager, professional, office worker & $22.2(1.7)$ & $5.9(2.6)$ & \\
\hline Service, sales & $13.8(1.5)$ & $15.0(4.0)$ & \\
\hline Manual worker & $22.4(1.7)$ & $15.9(3.7)$ & \\
\hline Unemployed & $41.6(1.9)$ & $63.2(5.2)$ & \\
\hline Living together, \% (SE) & $73.9(1.6)$ & $50.4(5.2)$ & $<0.01$ \\
\hline BMI, \% (SE) & & & 0.06 \\
\hline Under $18.5 \mathrm{~kg} / \mathrm{m}^{2}$ & $3.8(0.7)$ & $8.2(2.5)$ & \\
\hline Over $25 \mathrm{~kg} / \mathrm{m}^{2}$ & $33.1(1.9)$ & $34.7(4.3)$ & \\
\hline Current smoker, \% (SE) & $20.1(1.6)$ & $17.6(4.2)$ & 0.59 \\
\hline Heavy drinker, \% (SE) & $32.2(1.7)$ & $24.3(4.4)$ & 0.08 \\
\hline Adequate physical activity, \% (SE) & $14.7(1.3)$ & $8.3(2.9)$ & 0.10 \\
\hline Strength exercise, \% (SE) & $20.7(1.5)$ & $11.3(3.0)$ & 0.02 \\
\hline
\end{tabular}

SE: standard error, BMI: body mass index 
depression were significantly more frequent among individuals with suicidal ideation.

Table 3 presents the significant multivariable-adjusted odds ratios (ORs) with 95\% confidence intervals (CIs) of ICD-10 coded diseases and suicidal ideation. Notably, diagnoses of neoplasm (C00-C97) and mental disorder (F00-F99) were associated with the greatest increases in the probability of suicidal ideation [OR (95\% CI): 8.32 (1.01-68.56) and 6.19 (1.7222.33), respectively], although diseases of the musculoskeletal system and connective tissue (M00-M99) were also associated with suicidal ideation [OR (95\% CI): 1.93 (1.09-3.43)]. Conversely, injury, poisoning, and certain other consequences of

Table 2. Diseases diagnosed by primary care providers and the self-reported underlying disease among participants per suicidal ideation ( $\mathrm{N}=$ 1,211)

\begin{tabular}{|c|c|c|c|}
\hline & \multicolumn{2}{|c|}{ Suicidal ideation } & \multirow{2}{*}{ p-value } \\
\hline & No $(\mathrm{N}=1,057)$ & Yes $(\mathrm{N}=154)$ & \\
\hline \multicolumn{4}{|l|}{ Diseases diagnosed by primary care providers (ICD-10 codes) } \\
\hline Infectious and parasitic diseases (A00-B99) & $2.9(0.7)$ & $0.7(0.5)$ & 0.04 \\
\hline Neoplasm (C00-C97) & $0.5(0.3)$ & $1.5(1.4)$ & 0.34 \\
\hline Endocrine, nutritional, and metabolic diseases (E00-E90) & $4.8(0.7)$ & $4.2(2.5)$ & 0.81 \\
\hline Mental and behavioral disorders (F00-F99) & $1.2(0.4)$ & $10.6(3.7)$ & $<0.01$ \\
\hline Diseases of the nervous system (G00-G99) & $1.3(0.4)$ & $2.5(1,5)$ & 0.30 \\
\hline Diseases of the eye and adnexa (H00-H59) & $5.0(0.8)$ & $5.2(1.9)$ & 0.90 \\
\hline Diseases of the ear and mastoid process (H60-H95) & $2.1(0.7)$ & $1.7(1.1)$ & 0.80 \\
\hline Diseases of the circulatory system (I00-I99) & $13.1(1.3)$ & $12.9(3.4)$ & 0.96 \\
\hline Diseases of the respiratory system (J00-J99) & $28.4(1.8)$ & $25.4(4.5)$ & 0.52 \\
\hline Diseases of the digestive system (K00-K93) & $9.1(1.0)$ & $8.8(2.8)$ & 0.95 \\
\hline Diseases of the skin and subcutaneous tissue (L00-L99) & $6.0(0.9)$ & $8.2(3.0)$ & 0.44 \\
\hline Diseases of the musculoskeletal system and connective tissue (M00-M99) & $21.1(1.6)$ & $30.3(4.2)$ & 0.03 \\
\hline Diseases of the genitourinary system (N00-N99) & $3.1(0.6)$ & $3.2(2.0)$ & 0.96 \\
\hline Pregnancy, childbirth, and the puerperium (O00-O99) & $0.1(0.1)$ & N/A & \\
\hline Symptoms, signs and abnormal clinical and laboratory findings (R00-R99) & $3.0(0.6)$ & $4.4(1.8)$ & 0.41 \\
\hline Injury, poisoning, and certain other consequences of external causes (S00-T98) & $5.1(0.9)$ & $2.2(1.2)$ & 0.11 \\
\hline Factors influencing health status and contact with health services (Z00-Z99) & $9.7(1.2)$ & $7.0(2.4)$ & 0.35 \\
\hline \multicolumn{4}{|l|}{ Self-reported underlying diseases } \\
\hline Hypertension & $27.2(1.7)$ & $36.0(4.8)$ & 0.04 \\
\hline Diabetes mellitus & $10.3(0.9)$ & $11.5(2.8)$ & 0.68 \\
\hline Dyslipidemia & $18.6(1.4)$ & $24.8(4.0)$ & 0.11 \\
\hline Stroke & $2.4(0.6)$ & $6.7(2.2)$ & $<0.01$ \\
\hline Cardiovascular disease & $2.2(0.4)$ & $4.5(1.7)$ & 0.09 \\
\hline Depression & $6.0(0.9)$ & $23.4(4.1)$ & $<0.01$ \\
\hline Cancer & $5.3(0.8)$ & $4.5(1.7)$ & 0.68 \\
\hline
\end{tabular}

ICD: international classification of diseases, N/A: not applicable

Table 3. The association between the disease diagnosed by primary care providers and suicidal ideation

\begin{tabular}{llc}
\hline \multicolumn{1}{c}{ Diseases diagnosed by primary care providers (ICD-10 codes) } & OR (95\% CI) & p-value \\
\hline Neoplasm (C00-C97) & $8.32(1.01-68.56)$ & $<0.05$ \\
Mental and behavioral disorders (F00-F99) & $6.19(1.72-22.33)$ & $<0.01$ \\
Diseases of the musculoskeletal system and connective tissue (M00-M99) & $1.93(1.09-3.43)$ & 0.02 \\
Injury, poisoning, and certain other consequences of external causes (S00-T98) & $0.17(0.04-0.77)$ & 0.02 \\
\hline
\end{tabular}

Multiple logistic regression analyses were used to examine whether suicidal ideation was associated with specific diseases after controlling for age, sex, household income, education, occupation, marital status, smoking, alcohol consumption, and physical activity. ICD: international classification of diseases, OR: odds ratio, CI: confidence interval 
external causes (S00-T98) were associated with a lack of suicidal ideation [OR (95\% CI): 0.17 (10.04-0.77)]. We further calculated the frequency of visits to primary care providers during the most recent 2 -week period. In an analysis stratified by ICD-10 codes assigned by primary care providers, injury, poisoning, and certain other consequences of external causes were significantly more frequent among individuals without suicidal ideation $(\mathrm{p}<0.01)$. No significant associations were observed between other diseases diagnosed by primary care providers and the presence of suicidal ideation.

\section{DISCUSSION}

In this cross-sectional study of data from the KNHANES, we identified that approximately $12 \%$ of primary care patients reported suicidal ideation. This rate was higher than the range of $1.0-7.3 \%$ reported by a review of suicidal ideation among primary care patients in the U.S., ${ }^{15}$ and similar to the rates from German primary care studies in which the PHQ was used to assess suicidal ideation. ${ }^{16}$ According to another Korean study surveying patients visiting selected 15 primary care facilities in Seoul and Gyeonggi-do Provinces, approximately $18 \%$ of those reported suicidal ideation, ${ }^{17}$ which was higher than those of this representative study due to the difference of the living area and age distribution of the population. These figures are higher than those of the Korean general population, which was approximately 6\% in the 2013-2014 KNHANES, calculated using the question, "Have you ever seriously considered suicide in the last year?."18

The primary care patients with suicidal ideation in this study were more likely to be female, have a lower income and education level, live alone, and be unemployed, consistent with multiple reports describing risk factors for suicidal ideation. ${ }^{19-21}$ We further investigated potential differences in the chief primary care diagnoses of patients with and without suicidal ideation and found that the former were more likely to receive diagnoses of neoplasms (C00-C97), mental and/or behavioral disorders (F00-F99), and diseases of the musculoskeletal system and connective tissue (M00-M99), while the latter were more likely to be diagnosed with an injury, poisoning, and certain other consequences of external causes (S00-T98).

Previous studies have reported that primary care patients with suicidal ideation were more frequently diagnosed with depression $^{22}$ and anxiety disorders, ${ }^{23}$ compared to those without suicidal ideation. Consistent with those findings, we observed a higher frequency of diagnosed mental and behavioral disorders (F00-F99) among patients with suicidal ideation. This finding is highly plausible, as a previous systematic review of psychological autopsy studies on suicide reported that $>60 \%$ of suicidal deaths involved victims who had experienced a ma- jor depressive disorder or other mood disorders. ${ }^{24}$ Moreover, patients with neoplasms (C00-C97) have a high risk of psychological problems, ${ }^{25}$ consistent with our findings.

Additionally, we identified diseases of the musculoskeletal system and connective tissue (M00-M99) as primary causes of visits to primary care providers among individuals with suicidal ideation. Although the paucity of studies evaluated the association between musculoskeletal disorders and suicidal ideation, a review of the psychological risk factors associated with back and neck pain reported that these factors play an important role not only in chronic pain but also in the etiology of acute pain, particularly during the transition to chronic problems. ${ }^{26}$ Of musculoskeletal diseases, fibromyalgia, characterized by chronic, widespread musculoskeletal pain, ${ }^{27}$ is linked to depression and anxiety; indeed some authors have argued about the possibility to classify this syndrome into affective spectrum disorder. ${ }^{28}$ Accordingly, patients with physical pain due to musculoskeletal disorders may experience depressive symptoms, which would explain the observed significant association with suicidal ideation.

Our analysis revealed that injury, poisoning, and certain other consequences of external causes were diagnosed more frequently in patients without suicidal ideation. On the other hands, one previous study based on a Korean national health insurance database reported that patients who visited a medical facility for injury, poisoning, and certain other consequences of external causes (S00-T98) had a significantly increased risk of suicide death. ${ }^{29}$ This difference could be explained that severity of injury between primary care and emergency center. In the earlier study, disease information was solicited from all types of medical facilities, including psychiatric departments and emergency departments. In this study, however, we investigated only disease information from primary care patients with suicidal ideation, rather than suicidal death. Possibly, patients with injuries related to suicide attempts visited the emergency departments of general hospitals more frequently due to the severity of their conditions.

Currently, Korea reports the highest national frequency of physician visits worldwide. According to a 2017 OECD Health Statistics analysis, Korea reported the highest number of outpatient visits to physicians per capita among all OECD countries, with a rate of 16.0 per year in 2015 vs. the OECD average of 7.0 per year. ${ }^{30}$ Given that the frequent clinical visits made by Korean patients and the finding that patients with specific diseases face a higher risk of suicidal ideation support the suggestion that Korean physicians should screen high-risk patients for suicidal ideation. Accordingly, we recommend that primary physician should consider undergoing screening for suicidal ideation, when patients with low income (checking insurance information), living alone, or unemployed complain 
the symptom including mental or musculoskeletal disorders should consider undergoing screening for suicidal ideation.

This study had some limitations. First, the cross-sectional design did not allow us to confirm the events that occurred between suicidal ideation and visits to primary care providers within the 2-week period prior to the survey. Second, the assessment of suicidal ideation was based only on the PHQ-9. Therefore, we may have underestimated the frequency of suicidal ideation and could not evaluate suicidal plans or attempts. Third, the ICD-10 codes were based on the history of self-reported visits to primary care providers. Therefore, further research is needed to evaluate these associations in greater detail. Despite these limitations, our study was the first to examine the association of suicidal ideation with musculoskeletal diseases and mental disorders in a nationally representative sample of primary care patients in the country with the highest suicide rate worldwide.

In conclusion, the observed visit patterns and correlations of certain diagnoses with suicidal ideation suggest that primary care physicians should pay attention to patients with relevant disorders, consider screening suicidal ideation, and properly consult to a psychiatrist.

\section{Acknowledgments}

\section{None.}

\section{Conflicts of Interest}

The authors have no potential conflicts of interest to disclose.

\section{Author Contributions}

Conceptualization: Gyeongsil Lee, Jung-Ha Kim. Data curation: Gyeongsil Lee, Jung-Ha Kim. Formal analysis: Gyeongsil Lee, Jung-Ha Kim. Investigation: Gyeongsil Lee. Methodology: Jung-Ha Kim. Project administration: Jung-Ha Kim. Resources: Jung-Ha Kim. Software: Jung-Ha Kim. Supervision: Jung-Ha Kim. Validation: Jung-Ha Kim. Writing_original draft: Gyeongsil Lee. Writing—review \& editing: Gyeongsil Lee, Jung-Ha Kim.

\section{ORCID iDs}

Gyeongsil Lee

Jung-Ha Kim

https://orcid.org/0000-0003-1910-9658

https://orcid.org/0000-0002-7630-9501

\section{REFERENCES}

1. Weiland M. Geography of loss. Science 2019;365:736-737.

2. Korea Suicide Rrevention Center. 2018 Statistical Data of Korean Suicide. Available at: https://www.statista.com/statistics/789337/southkorea-suicide-death-rate/. Accessed March 30, 2020.

3. World Health Organization. Public Health Action for the Prevention of Suicide: A Framework. Available at: https://www.who.int/mental_ health/publications/prevention_suicide_2012/en/. Accessed September 20, 2018.

4. Public Health Service. The Surgeon General's Call to Action to Prevent Suicide. Available at: https://www.sprc.org/resources-programs/surgeon-generals-call-action-prevent-suicide. Accessed September 20, 2018.

5. U.S. Surgeon General and of the National Action Alliance for Suicide Prevention. 2012 National Strategy for Suicide Prevention: Goals and Objectives for Action. Washington, DC: Dept. of Health and Human
Services, U.S Public Health Service; 2012.

6. Bunney W, Kleinman A, Pellmar T, Goldsmith S. Reducing Suicide: A National Imperative. Washington (DC): National Academies Press; 2002.

7. Luoma JB, Martin CE, Pearson JL. Contact with mental health and primary care providers before suicide: a review of the evidence. Am J Psychiatry 2002;159:909-916.

8. Jordan P, Shedden-Mora MC, Löwe B. Predicting suicidal ideation in primary care: An approach to identify easily assessable key variables. Gen Hosp Psychiatry 2018;51:106-111.

9. USPSTF. Suicide Risk in Adolescents, Adults and Older Adults: Screening. Published 2014. Available at: https://www.uspreventiveservicestaskforce.org/Page/Document/UpdateSummaryFinal/suicide-risk-inadolescents-adults-and-older-adults-screening. Accessed Sep 20, 2018.

10. Kweon S, Kim Y, Jang MJ, Kim Y, Kim K, Choi S, et al. Data resource profile: the Korea national health and nutrition examination survey (KNHANES). Int J Epidemiol 2014;43:69-77.

11. Kroenke K, Spitzer RL, Williams JB. The PHQ-9: validity of a brief depression severity measure. J Gen Intern Med 2001;16:606-613.

12. Han C, Jo SA, Kwak JH, Pae CU, Steffens D, Jo I, et al. Validation of the Patient Health Questionnaire-9 Korean version in the elderly population: the Ansan Geriatric study. Compr Psychiatry 2008;49:218-223.

13. World Health Organization. The Asia-Pacific Perspective: Redefining Obesity and Its Treatment. Sydney: Health Communications Australia; 2000.

14. Hagströmer M, Oja P, Sjöström M. The International Physical Activity Questionnaire (IPAQ): a study of concurrent and construct validity. Public Health Nutr 2006;9:755-762.

15. Schulberg HC, Bruce ML, Lee PW, Williams Jr JW, Dietrich AJ. Preventing suicide in primary care patients: the primary care physician's role. Gen Hosp Psychiatry 2004;26:337-345.

16. Wiborg JF, Gieseler D, Löwe B. Suicidal ideation in German primary care. Gen Hosp Psychiatry 2013;35:366-369.

17. Choi YJ, Lee WY. The prevalence of suicidal ideation and depression among primary care patients and current management in South Korea. Int J Ment Health Syst 2017;11:18.

18. Ministry of Health \& Welfare, Korea Suicide Prevention Center. 2019 White Book for Suicide Prevention. Published 2019. Available at: http:// spckorea-stat.or.kr/boadpublishview.do. Accessed March 30, 2020.

19. Brown GK, Beck AT, Steer RA, Grisham JR. Risk factors for suicide in psychiatric outpatients: a 20-year prospective study. J Consult Clin Psychol 2000;68:371-377.

20. Nock MK, Borges G, Bromet EJ, Alonso J, Angermeyer M, Beautrais A, et al. Cross-national prevalence and risk factors for suicidal ideation, plans and attempts. Br J Psychiatry 2008;192:98-105.

21. Chin YR, Lee HY, So ES. Suicidal ideation and associated factors by sex in Korean adults: a population-based cross-sectional survey. Int J Public Health 2011;56:429-439.

22. Schulberg HC, Lee PW, Bruce ML, Raue PJ, Lefever JJ, Williams JW Jr, et al. Suicidal ideation and risk levels among primary care patients with uncomplicated depression. Ann Fam Med 2005;3:523-528.

23. Bomyea J, Lang AJ, Craske MG, Chavira D, Sherbourne CD, Rose RD, et al. Suicidal ideation and risk factors in primary care patients with anxiety disorders. Psychiatry Res 2013;209:60-65.

24. Cavanagh JT, Carson AJ, Sharpe M, Lawrie SM. Psychological autopsy studies of suicide: a systematic review. Psychol Med 2003;33:395-405.

25. Krebber A, Buffart L, Kleijn G, Riepma IC, de Bree R, Leemans CR, et al. Prevalence of depression in cancer patients: a meta-analysis of diagnostic interviews and self-report instruments. Psychooncology 2014;23: 121-130.

26. Linton SJ. A review of psychological risk factors in back and neck pain. Spine 2000;25:1148-1156.

27. Bair MJ, Krebs EE. Fibromyalgia. Ann Intern Med 2020;172:ITC33ITC48.

28. Aguglia A, Salvi V, Maina G, Rossetto I, Aguglia E. Fibromyalgia syndrome and depressive symptoms: Comorbidity and clinical correlates. 
J Affect Disord 2011;128:262-266.

29. Choi SB, Lee W, Yoon JH, Won JU, Kim DW. Ten-year prediction of suicide death using Cox regression and machine learning in a nationwide retrospective cohort study in South Korea. J Affect Disord 2018;231:8-
14.

30. Ministry of Health and Welfare and Korea Institute for Health and Social Affairs. OECD Health Statics 2017. Sejong-si: Kyungsung Media; 2017. 